\title{
On the Binding of Congo Red to Amyloid Fibrils
}

\author{
Alba Espargaró, ${ }^{\#[a, b]}$ Salomé Llabrés, ${ }^{\#[c]}$ Sven J Saupe, ${ }^{[\mathrm{d}]}$ Carles Curutchet, ${ }^{[[a, e]} \mathrm{F}$. Javier Luque, ${ }^{*[\mathrm{e}, \mathrm{f}, \mathrm{g}]}$ \\ and Raimon Sabaté ${ }^{\star[a, b]}$
}

\begin{abstract}
Amyloids are characterized by their capacity to bind Congo Red (CR), one of the most used amyloid specific dyes. The structural features of $\mathrm{CR}$ binding have been unknown for years, mainly because of the lack of amyloid structures solved at high resolution. In the last few years, solid state NMR has allowed to decipher the structural features of amyloids, such as the HET-s prion forming domain (HET-S PFD), which also has recently been used to assay the amyloid-CR interface at atomic resolution. Here, we combine spectroscopic data with molecular docking, molecular dynamics, and excitonic quantum/molecular mechanics calculations to examine and rationalize CR binding to amyloids. In contrast to a previous assumption on the binding mode, our results suggest that CR binding to the HET-S PFD involves a cooperative process entailing the formation of a complex with 1:1 stoichiometry. This provides a molecular basis to explain the bathochromic shift in the maximal absorbance wavelength when $\mathrm{CR}$ is bound to amyloids.
\end{abstract}

Amyloids are fibrillary protein polymers displaying a cross $\beta$-structure in which individual $\beta$-strands run perpendicular to the fibril axis,$^{[1]}$. Amyloid fibrils are thermodynamically more stable than the native, non-aggregated proteins. The formation of amyloids occurs in many branches of life as amyloid aggregation has been described from mammals to fungi and bacteria, and can be either a pathological or a functional process, ${ }^{[2]}$. In humans, amyloid aggregation is related to up to thirty-six degenerative protein misfolding disorders, including nonneurologic and neurologic illnesses, such as type 2 diabetes or Alzheimer's and Parkinson's diseases. ${ }^{[1 a, 3]}$

Amyloids are generally examined by exploiting their ability to bind Congo Red (CR), one of the most widely used amyloid specific dyes. ${ }^{[4]}$ CR $\left(3,3^{\prime}-\left(\left[1,1^{\prime}\right.\right.\right.$-biphenyl]-4,4'-diyl)bis(4aminonaphthalene-1-sulfonic acid; Figure 1 ) is a long and flat

[a] Dr. A. Espargaró, Prof. Dr. C. Curutchet and Prof. Dr. R. Sabaté Department of Pharmacy and Pharmaceutical Technology and Physical-Chemistry, School of Pharmacy and Food Sciences, University of Barcelona

Joan XXIII, 27-31, Barcelona, E-08028 (Spain)

E-mail: rsabate@ub.edu

[b] Dr. A. Espargaró and Prof. Dr. R. Sabaté

Institute of Nanoscience and Nanotechnology $\left(\mathrm{IN}^{2} \mathrm{UB}\right)$

[c] Dr. Salomé Llabrés

School of Chemistry, University of Edimburgh

David Brewster Road, EH9 3FJ, Edinburgh (UK)

[d] Dr. Sven J. Saupe

Institut de Biochimie et de Génétique Cellulaire, UMR 5095, CNRS,

Université de Bordeaux

1 rue Camille St Saens, Bordeaux, 33077 (France)

[e] Prof. Dr. C. Curutchet and Prof. Dr. F. J. Luque

Institute of Theoretical and Computational Chemistry (IQTCUB)

[f] Prof. Dr. F. J. Luque

Department of Nutrition, Food Sciences, and Gastronomy, School of

Pharmacy and Food Sciences, University of Barcelona

Prat de la Riba 171, Santa Coloma de Gramenet, E-08921 (Spain)

[g] Prof. Dr. F. J. Luque

Institute of Biomedicine (IBUB)

Co-authors

Corresponding authors

Supporting information for this article is given via a link at the end of the document. azo dye, formed by an apolar region, which is defined by the central biphenyl group linked through diazo units to flanking naphthalene rings, and a polar region, which contains an amino group and a negatively charged sulphate group bound to each naphthalene ring. Several binding modes were proposed for the interaction of $\mathrm{CR}$ with amyloids, ${ }^{[5]}$ but the fine structural details remained elusive due to the lack of high-resolution amyloid structures. In the last few years, solid state NMR (ssNMR) has allowed to determine amyloid structures at atomic resolution, ${ }^{[6]}$ as exemplified by the case of the HET-s prion forming domain (HET-s PFD). Beyond the role in cell death activation, ${ }^{[7]} \mathrm{HET}-\mathrm{s}$ is a well-studied system to elucidate the action of prions and amyloid formation. ${ }^{[8]}$ Indeed, HET-s PFD was one of the first completed amyloid structures. ${ }^{[9]}$ This structure has been used by Schütz and co-workers to describe the amyloid-CR interface at atomic resolution. ${ }^{[10]}$ In particular, the analysis of chemical-shift perturbations triggered upon $\mathrm{CR}$ binding in two-dimensional ${ }^{13} \mathrm{C}-$ ${ }^{13} \mathrm{C}$ correlation spectra of stained and unstained $\left[{ }^{13} \mathrm{C},{ }^{15} \mathrm{~N}\right]-$ labelled fibrils associated to polarization-transfer experiments revealed the protein surface that interacts with $\mathrm{CR}$ molecules. ${ }^{[10]}$ Strong polarization transfer was observed from $C R$ to residues S227, A228, K229, S263 and E265, which are clustered in the vicinity of the two-residue arc connecting $\beta$-sheets $\beta 1 \mathrm{a} / \beta 1 \mathrm{~b}$ and $\beta 3 a / \beta 3 a$ in HET-s PFD. ${ }^{[9,10]}$ The results led to a structural model where CR molecules are aligned with their long axis parallel to the fibril axis. The proposed binding mode would be compatible with a 3:1 stoichiometry, wherein three HET-s PFD monomers in a left-handed $\beta$-solenoid, with each molecule forming two helical windings, would be necessary to bind a CR molecule.

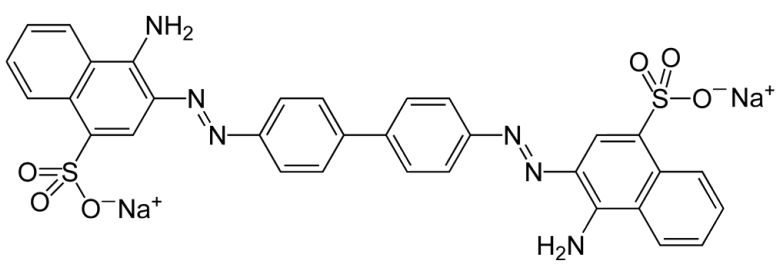

Figure 1. Structure of Congo red (CR).

On the basis of this structural model, we carried out a detailed spectroscopic analysis to gain insight into the general mechanism of CR binding to the HET-S PFD. As shown in Figure 2 (see also Supporting Information Figures S1-S3), a typical binding assay (see Experimental Section) was performed to determine the spectra of free and bound $C R$, and the binding constant $\left(K_{\mathrm{d}}\right)$ and maximal binding $\left(B_{\max }\right)$, thus defining the stoichiometry of the complex ( $n$ ). Maximal absorption wavelengths of 490 and $521 \mathrm{~nm}$ were obtained for the free and bound CR, respectively, revealing a shift of $31 \mathrm{~nm}$ between the wavelength of both maxima upon $\mathrm{CR}$ binding. The differential spectrum shows the typical maximum at $541 \mathrm{~nm}$ indicative of $C R$ binding to amyloid structures (Figure 2). Values of $21.64 \pm 1.40$ 
$\mu \mathrm{M}, 10.38 \pm 0.38 \mu \mathrm{M}$ and $1.43 \pm 0.06$ were determined for $K_{\mathrm{d}}, B_{\max }$ and Hill coefficient $(h)$, respectively, at $10 \mu \mathrm{M}$ of HET-s PFD. Furthermore, the data showed a stoichiometry of $n=1$ (CR-HETs PFD), which differs from the expected value $(n=3) \cdot{ }^{[8]}$ In addition, the Hill coefficient suggested a cooperative binding, which cannot be explained by the structural model proposed by Schütz et al., where CR molecules are aligned along the fibril groove axis. ${ }^{[8]}$ Since more than one CR molecule could be cooperatively fixed at the HET-s PFD structure, we performed an exhaustive sampling of alternative binding modes by combining docking and molecular dynamics (MD) simulations with excitonic quantum/ molecular mechanics (QM/MM) calculations with the aim to rationalize the experimental data determined for the $\mathrm{CR}$ binding to HETs PFD. These studies led to an alternative binding mode in the fibril groove, but entailing a stoichiometry of $n=1$, in agreement with spectroscopic data (Supporting Information Figure S4). In the same direction, it has been recently shown that non-covalent, electrostatic interactions induce positive cooperative binding of small molecules to Alzheimer's and Parkinson's disease-related amyloids. ${ }^{[11]}$
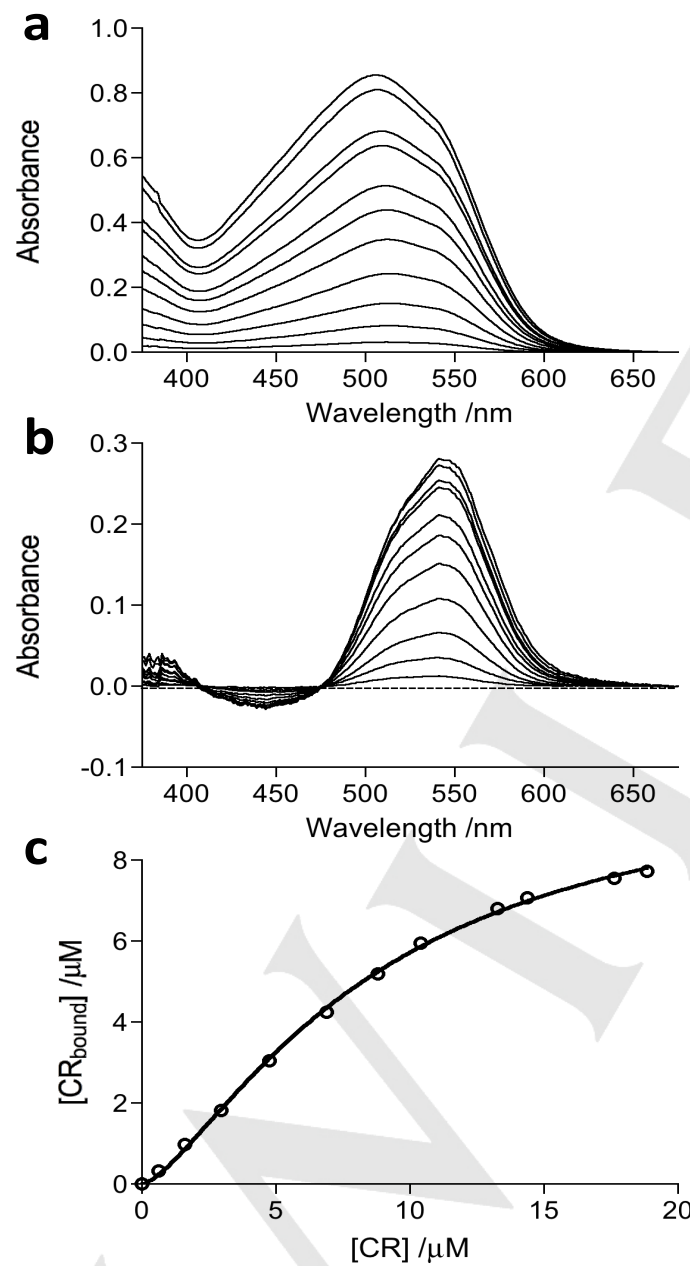

Figure 2. Spectral changes on the CR Binding to HET-s PFD fibrils. a) Visible absorption spectra of increasing concentration of CR in the presence of $10 \mu \mathrm{M}$
HET-s PFD fibrils. b) Differential spectra of the CR-HET-s PFD complex and free CR. c) CR-HET-s PFD binding saturation curve.

The structural model proposed for the $1: 1$ stoichiometric binding of CR to HET-s is shown in Figure 3. This binding mode was stable in three independent MD simulations (see Supporting Information Figures S5-S8). The binding mode involves the electrostatic interaction of one of the sulphate groups with the protonated amino of $\mathrm{K} 229$ in monomer $i$, and the other sulphate with the guanidinium group of R225 in monomer $i+2$ (average distances of $2.80 \pm 1.68$ and $3.85 \pm 1.17 \AA$, respectively, as determined from the analysis of three independent MD replicas, although a skewed distribution of distances was found). These interactions are enabled through a regular arrangement where the long axis of $\mathrm{CR}$ deviates around $20^{\circ}$ from the axis of the fibril groove. In turn, this favours the formation of $\pi$-stacking interactions between the aromatic moieties of naphthalene and biphenyl rings of neighbouring CR molecules (average distance of $4.04 \pm 0.39 \AA$ between the centre-of-mass of naphthalene and biphenyl rings of $C R$ molecules $k$ and $k+1$, respectively; Figure S8). Additionally, the amino moieties can establish hydrogen bonds alternatively with the side-chains of S227 and S263 in monomer $i$. Noteworthy, this structural model agrees with the strong polarization-transfer peaks experimentally observed between CR and residues S227, A228, K229, S263 and E265 ${ }^{[10]}$ as noted by the distances between the CR hydrogen atoms and the carbon atoms of the protein residues (Supporting Information Figure S9 and Table S2). On the other hand, the parallel arrangement of the stacked CR molecules also favours the adoption of a more planar structure, as the population of planar conformations ( $0 \pm 15$ degrees) around the dihedral angle of the biphenyl moiety increases from $12 \%$ in water solution to $39 \%$ in the CR-bound fibril model according to the sampling obtained from MD simulations.
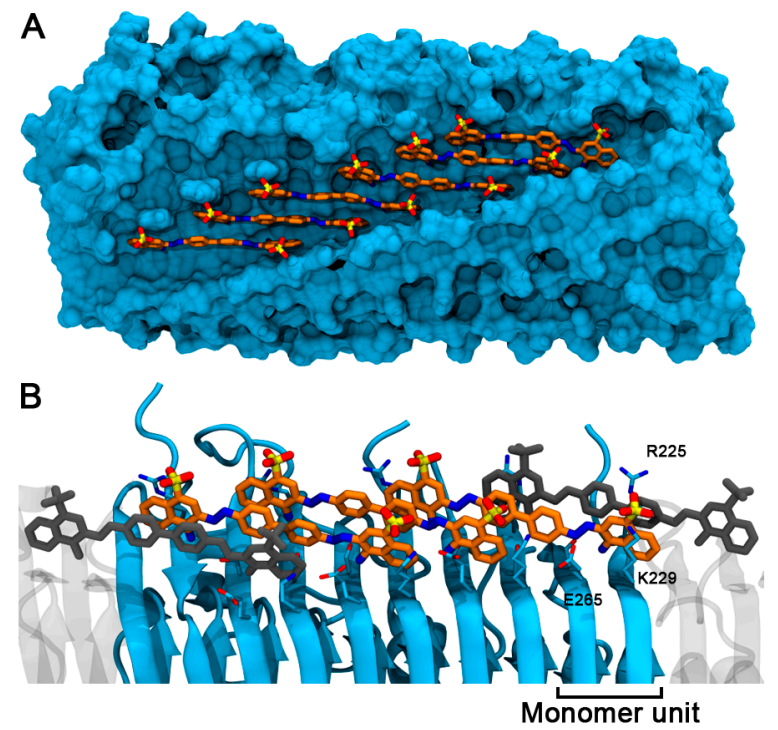

Figure 3. (A) Representation of the proposed 1:1 stoichiometric binding of CR to HET-s PFD fibrils. Six stacked CR molecules (orange sticks) bind to the arc 
connecting $\beta$-sheets $\beta 1 \mathrm{a} / \beta 1 \mathrm{~b}$ and $\beta 3 \mathrm{a} / \beta 3 \mathrm{a}$ in HET-s PFD shown as blue surface. (B) Interactions of CR molecules with K229 and R226 of two consecutive monomer units. Two $\mathrm{CR}$ molecules and two monomers are shown in grey at each end of the fibril to highlight the continuity of this binding mode along the length of the fibril.

To confirm this binding mode, we determined the theoretical spectrum of $\mathrm{CR}$ in its monomeric and aggregated (trimer and hexamer) species in solution and HET-s bound states, respectively, by performing excitonic $\mathrm{QM} / \mathrm{MM}$ calculations over structures sampled along the MD trajectories. Excitonic effects were accounted for in the simulation of the spectra by computing the electronic couplings among $\mathrm{CR}$ monomers in the bound state. As shown in Figure 4 (see also Supporting Information Figures S10-S12), the spectral reconstruction supports the hypothesis that CR binds to HET-s PFD following a cooperative mechanism. Importantly, the bathochromic shift of bound $C R$ $(\sim 12 \mathrm{~nm})$ could only be explained by several CR monomers coordinately fixed to HET-s PFD fibrils, enabling also to dissect the contribution of distinct effects to the induction of the red shift (Supporting Information Figure S12). First, the (uncoupled) electronic transition energies of the six CR molecules are shifted when bound to the fibrils due to the constrained conformation of the stacked CRs $(+22 \mathrm{~nm})$ and the establishment of dye-protein $(-11 \mathrm{~nm})$ and dye-dye $(+3 \mathrm{~nm})$ interactions. Interestingly, the larger contribution of the conformational restraint relative to the dye-protein interactions may explain why a similar CR spectrum is observed upon binding to different amyloids.

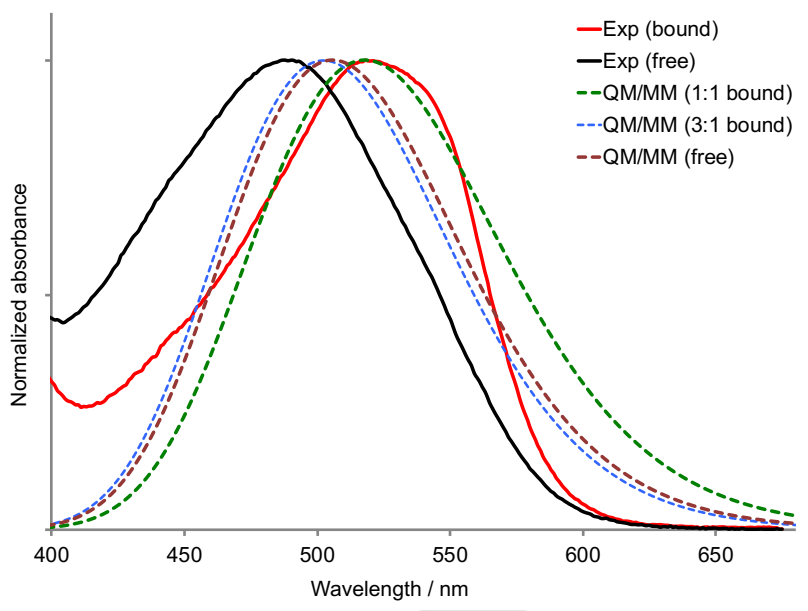

Figure 4. Experimental and theoretical TD-DFT absorption spectra of CR in water and bound to HET-s PFD fibrils.

A further shift, however, can arise from the strong excitonic coupling among CR molecules, estimated to be around $\sim 110$ $\mathrm{cm}^{-1}$ for nearest-neighbour interactions (see Supporting Information Tables S3-S4). Our analysis, however, points to a minor impact of excitonic effects on the shift $(-2 \mathrm{~nm})$, thus indicating that the $\mathrm{CR}$ molecules do not behave as a Jaggregate, a term used to describe the observation of excitonic bathochromic shifts due to the aggregation of dyes. ${ }^{[10]}$ This behaviour occurs when excitonic coupling delocalizes the excited states along several molecules leading to a redistribution of the oscillator strength to the red edge. In our simulations, however, the net redistribution of oscillator strength does not lead to an overall shift of the spectrum (Supporting Information Figure S13). Finally, we note that the theoretical spectrum does not include vibronic effects, which could explain the shoulder at $\sim 540 \mathrm{~nm}$ observed experimentally but absent in our simulations, as we assume a Gaussian line-shape. Such effects could also be partially responsible for the underestimation of the bathochromic shift, as the position of the maximum depends on the shape of the band.

In contrast with the results obtained for the 1:1 structural model, the binding mode corresponding to a 3:1 stoichiometry showed a larger structural destabilization, as noted in larger fluctuations of the RMSD profiles for the CR molecules, and larger distances from the sulphate groups to K229 residues (Supporting Information Figure S9-S11). Furthermore, QM/MM calculations predicted that this binding model leads to a negligible shift on the spectra compared to our simulation in water (Figure 4).

Overall, data presented here provides a novel view about the binding mechanism that underlies the labelling of HET-S PFD fibrils by $C R$. The results suggest that $C R$ bound to amyloid fibrils is oriented with the long axis oblique to the fibril, characterized by an angular deviation close to $20^{\circ}$, which permits the mutual interaction between the aromatic moieties of vicinal CR molecules. This arrangement provides a molecular basis to justify the cooperative association of $\mathrm{CR}$ molecules along the fibril axis in the fibrillar microenvironment, and the constrained conformational flexibility can be interpreted as the main contribution to the bathochromic shift of the maximal absorbance wavelength when $\mathrm{CR}$ is bound to amyloids, a criteria broadly accepted in studies of amyloidogenic aggregation processes. Finally, since CR binds to most amyloids displaying a bathochromic shift, birefringence under polarized light, dichroism and fluorescence, ${ }^{[4 c, 12]}$ present results permit to envisage that such cooperative binding mechanism may be involved in the binding of CR to amyloid fibrils.

\section{Experimental Section}

$\mathrm{CR}$ and other reagents were obtained from Sigma. CR was solubilized in miliQ water (Millipore) at $10 \mathrm{mM}$. HET-s PFD protein expressed as a Cterminal histidine-tagged construct in Escherichia coli was purified under denaturing conditions by affinity chromatography. Buffer was exchanged for $175 \mathrm{mM}$ acetic acid. $\mathrm{pH} 7$ bundles fibrils were formed in 1:1 mixture of $175 \mathrm{mM}$ acetic acid and $1 \mathrm{M}$ Tris $\cdot \mathrm{HCl} \mathrm{pH} 8$ at $37^{\circ} \mathrm{C}$ and $1400 \mathrm{rpm}$ for $24 \mathrm{~h}$ using a Thermomixer (Eppendorf) and a protein concentration of $100 \mu \mathrm{M}$.

The binding of CR to HET-s PFD fibrils was tracked by recording the absorbance spectra from 375 to $675 \mathrm{~nm}$. To detect the typical amyloid band at $541 \mathrm{~nm}$, differential CR spectra in the presence and absence of protein were used. Spectra were fitted to four overlapping Gaussian curves. The amplitude, centre, full width at half maximum (FWHM) and area of each Gaussian function were calculated. HET-s PFD-CR binding was analysed using a saturation binding equation: $\boldsymbol{Y}=$ 
$\boldsymbol{B}_{\max } \cdot \boldsymbol{x}^{h} /\left(\boldsymbol{K}_{\boldsymbol{d}}+\boldsymbol{x}^{h}\right)$. This expression describes the binding of a ligand to a receptor that follows the law-of-mass action. $\boldsymbol{B}_{\max }$ is the maximal binding, $\boldsymbol{x}$ is the CR concentration, $\boldsymbol{K}_{\boldsymbol{d}}$ is the concentration of ligand required to reach half-maximal binding and $\boldsymbol{h}$ is the Hill coefficient.

Docking calculations were performed using rDOCK software ${ }^{[13]}$ on two protein models of the HETs amyloid: the sSNMR structure obtained by Wasmer et al. ${ }^{[9]}$ (PDB id 2RNM; note that the protein fold is very similar to the ssNMR structure with PDB code $2 \mathrm{KJ} 3)^{[14]}$ and the protein model used by Schultz et al. ${ }^{[10 a]}$ (PDB id 2LBU). The stability and properties of the two proposed binding modes (representative of $3: 1$ and 1:1 stoichiometries) were studied by means of MD simulations. A fully atomistic description of a decamer of HET-s PDF, the CR molecules and the solvent was used (See Supporting Information for details). Three 50ns-long independent trajectories for each studied system, and a negative control of a single molecule of $\mathrm{CR}$ in water (100 ns; used for excitonic QM/MM computations) were obtained using the pmemd module of the AMBER14 package. ${ }^{[15]}$

The absorption properties of bound and free CR were calculated through polarizable quantum/molecular mechanics (QM/MMPol) excited state calculations. ${ }^{[16]}$ Excited-states were described either using Zerner's intermediate neglect of differential overlap (ZINDO) semi-empirical method ${ }^{[17]}$ and time-dependent density functional theory (TD-DFT) based on the long-range corrected CAM-B3LYP functional ${ }^{[18]}$ and the $6-31 \mathrm{G}(\mathrm{d})$ basis set, whereas the environment (protein, water and other $C R$ molecules) was described using the Amber ff12pol AL force field. ${ }^{[19]}$ In order to dissect the origin of the solvatochromic shifts, we also performed calculations on the CRs as found in the 1:1 bound structure including only the water or the water+CR environment. In these calculations, the water solvent was described using a continuum model through the threelayer QM/MMPol/ddCOSMO implementation, based on the recently developed linear-scaling domain decomposition algorithm for COSMO. ${ }^{[20]}$ Exciton couplings between $\mathrm{CR}$ molecules were computed considering both Coulomb and environment screening terms from the transition densities obtained in the QM/MMPol calculations. ${ }^{[5]}$ All QM/MMPol calculations were performed using a locally modified version of Gaussian09. ${ }^{[21]}$ Absorption spectra were modelled assuming a Gaussian line-shape with $\mathrm{FWHM}$ of $0.5 \mathrm{eV}$ from the QM/MMpol data (transition energies and couplings) averaged along 100 structures extracted from the MD trajectories. Excitonic effects were taken into account by diagonalizing the excitonic Hamiltonian built from the $C R$ energies (diagonal elements) and their electronic couplings (off-diagonal elements). The impact of static disorder was explored by averaging homogeneous spectra over a random Gaussian distribution of the site energies of CR molecules. Several tests, however, indicated that disorder does not affect the absorption wavelength predicted for the exciton bands, although it impacts somewhat their relative intensities.

\section{Acknowledgements}

This work was supported by the Ministerio de Economía y Competitividad (MINECO/AEI/FEDER: CTQ2017-88446-R, SAF2014-57094-R, SAF2017-88107-R, CTQ2017-89924-P, MDM-2017-0767) and Generalitat de Catalunya (GC: 2014SGR938, 2017SGR1746). C.C. is a Serra Húnter fellow (GC). The Consorci de Serveis Universitaris de Catalunya
(CSUC; Molecular Recognition project) is acknowledged for computational resources.

Keywords: Congo red $\cdot$ Amyloids $\cdot$ prions $\cdot$ proteins $\bullet$ dye binding

[1] a) F. Chiti, C. M. Dobson, Annu Rev Biochem 2006, 75, 333-366; b) D. Eisenberg, R. Nelson, M. R. Sawaya, M. Balbirnie, S. Sambashivan, M. I. Ivanova, A. O. Madsen, C. Riekel, Acc Chem Res 2006, 39, 568-575.

[2] a) T. R. Jahn, S. E. Radford, Arch Biochem Biophys 2008, 469, 100-117; b) F. Rousseau, J. Schymkowitz, L. Serrano, Curr Opin Struct Biol 2006, 16, 118-126

[3] a) R. R. Kopito, D. Ron, Nat Cell Biol 2000, 2, E207-209; b) J. D. Sipe, M. D. Benson, J. N. Buxbaum, S. I. Ikeda, G. Merlini, M. J. Saraiva, P Westermark, Amyloid 2016, 23, 209-213.

[4] a) P. Westermark, M. D. Benson, J. N. Buxbaum, A. S. Cohen, B. Frangione, S. Ikeda, C. L. Masters, G. Merlini, M. J. Saraiva, J. D. Sipe, Amyloid 2007, 14, 179-183; b) D. P. Steensma, Arch Pathol Lab Med 2001, 125, 250-252; c) W. E. Klunk, R. F. Jacob, R. P. Mason, Anal Biochem 1999, 266, 66-76.

[5] a) H. Inouye, J. T. Nguyen, P. E. Fraser, L. M. Shinchuk, A. B. Packard, D. A. Kirschner, Amyloid 2000, 7, 179-188; b) H. LeVine, 3rd, Amyloid 2005, $12,5-14$; c) C. Wu, Z. Wang, H. Lei, W. Zhang, Y. Duan, J Am Chem Soc 2007, 129, 1225-1232.

[6] A. Espargaro, M. A. Busquets, J. Estelrich, R. Sabate, Int J Nanomedicine 2015, 10, 6975-6983.

[7] a) C. Ritter, M. L. Maddelein, A. B. Siemer, T. Luhrs, M. Ernst, B. H. Meier, S. J. Saupe, R. Riek, R. Nature 2005, 435, 844-848. b) R. Riek, S. J. Saupe. Cold Spring Harb Pespect Biol 2016, 8, a023515.

[8] a) J. Dolenc, B. H. Meier, V. H. Rusu, W. F. van Gunsteren. Phys Chem Chem Phys 2016, 18, 5860-5866. b) A. A. Smith, F. Ravotti, E. Testori, R. Cadalbert, M. Ernst, A. Bockmann, B. H. Meier. J Biomol NMR. 2017, 67, 109-119. c) M. A. Walti, T. Schmidt, D. T. Murray, H. B. Wang, J. E. Hinshaw, G. M. Clore. Proc Natl Acad Sci USA 2017, 114, 9104-9109. d) A. A. Smith, M. Ernst, S. Riniker, B. H. Meier. Angew Chem Int Ed 2019, 58, 9383-9388.

[9] C. Wasmer, A. Lange, H. Van Melckebeke, A. B. Siemer, R. Riek, B. H. Meier, Science 2008, 319, 1523-1526.

[10] a) A. K. Schutz, A. Soragni, S. Hornemann, A. Aguzzi, M. Ernst, A Bockmann, B. H. Meier, Angew Chem Int Ed 2011, 50, 5956-5960; b) C. Gowda, G. Zandomeneghi, H. Zimmermann, A.K. Schütz, A. Böckmann, M. Ernst, B. H. Meier, J Biomol NMR 2017, 69, 207-213

[11] J. L. Cifelli, C. C. Capule, J. Yang, ACS Chem Neurosci 2019, 10, 991995.

[12] a) R. P. Linke, Virchows Arch 2000, 436, 439-448; b) W. E. Klunk, R. F. Jacob, R. P. Mason, Methods Enzymol 1999, 309, 285-305; c) M. R. Nilsson, Methods 2004, 34, 151-160.

[13] S. Ruiz-Carmona, D. Alvarez-García, N. Foloppe, A. B. Garmendia-Doval S. Juhos, P. Schmidtke, X. Barril, R. E. Hubbard, S. D. Morley. PLoS Comput. Biol. 2014, 10, e1003571.

[14] H. van Melckebeke, C. Wasmer, A. Lange, E. AB, A. Loquet, A Böckmann, B. H. Meier. J. Am. Chem. Soc. 2010, 132, 13765-13775.

[15] D. A. Case, V. Babin, J. T. Berryman, R. M. Betz, Q. Cai, D. S. Cerutti., T. E. Cheatham III, T. A. Darden, R. E. Duke, H. Gohlke, A. E. Goetz, et al., University of California, San Francisco, 2014.

[16] C. Curutchet, A. Munoz-Losa, S. Monti, J. Kongsted, G. D. Scholes, B. Mennucci, J Chem Theory Comput 2009, 5, 1838-1848.

[17] M. C. Zerner, in In Reviews of Computational Chemistry, Vol. 2 (Eds.: K. B. Lipkowitz, D. B. Boyd), VCH, New York, 1991, pp. 313-366.

[18] T. Yanai, D. P. Tew, N. C. Handy, Chem. Phys. Lett. 2004, 393, 51-57.

[19] a) J. Wang, P. Cieplak, J. Li, Q. Cai, M. Hsieh, H. Lei, R. Luo, Y. Duan, J Phys Chem B 2011, 115, 3100-3111; b) J. Wang, P. Cieplak, J. Li, T. Hou, R. Luo, Y. Duan, J Phys Chem B 2011, 115, 3091-3099.

[20] F. Lipparini, G. Scalmani, L. Lagardère, B. Stamm, E. Cancès, Y. Maday, J. P. Piquemal, M. J. Frisch, B. Mennucci, J. Chem. Phys. 2014, 141, 184108.

[21] M. J. Frisch, G. W. Trucks, H. B. Schlegel, G. E. Scuseria, M. A. Robb, J. R. Cheeseman, G. Scalmani, V. Barone, B. Mennucci, G. A. Petersson, et al., revision A.2 ed., Gaussian, Inc., 2009. 
Entry for the Table of Contents (Please choose one layout)

Layout 1:

\section{COMMUNICATION}

Cooperative labelling of amyloids: CR bound to amyloid fibrils is oriented with the long axis oblique to the fibril axis and the plane radial to the fibril core, following a cooperative mechanism characterized by a $1: 1$ stoichiometric complex.

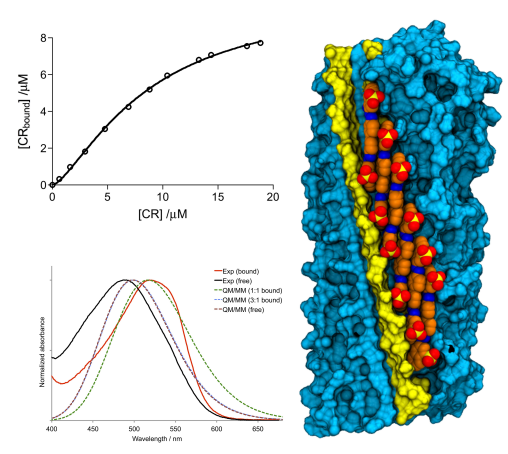

Alba Espargaró, ${ }^{\#[a, b]}$ Salomé Llabrés, ${ }^{[[c]}$ Sven J Saupe, ${ }^{[d]}$ Carles Curutchet, ${ }^{*}[a, e]$ F. Javier Luque, ${ }^{*}[e, f, g]$ and Raimon Sabaté ${ }^{*[a, b]}$

Page No. - Page No.

On the Binding of Congo red to Amyloid Fibrils 